\title{
Crystal structure of the primary piRNA biogenesis factor Zucchini reveals similarity to the bacterial PLD endonuclease Nuc
}

\author{
FRANKA VOIGT, ${ }^{1}$ MICHAEL REUTER, ${ }^{2,3}$ ANISA KASARUHO, ${ }^{2,3}$ EIKE C. SCHULZ, ${ }^{1}$ RAMESH S. PILLAI, ${ }^{2,3,4}$ \\ and ORSOLYA BARABAS ${ }^{\mathbf{1 , 4}}$ \\ ${ }^{1}$ European Molecular Biology Laboratory, 69117 Heidelberg, Germany \\ ${ }^{2}$ European Molecular Biology Laboratory, 38042 Grenoble, France \\ ${ }^{3}$ CNRS-UJF-EMBL International Unit (UMI 3265) for Virus Host Cell Interactions (UVHCI), 38042 Grenoble, France
}

\begin{abstract}
Piwi-interacting RNAs (piRNAs) are a gonad-specific class of small RNAs that associate with the Piwi clade of Argonaute proteins and play a key role in transposon silencing in animals. Since biogenesis of piRNAs is independent of the doublestranded RNA-processing enzyme Dicer, an alternative nuclease that can process single-stranded RNA transcripts has been long sought. A Phospholipase D-like protein, Zucchini, that is essential for piRNA processing has been proposed to be a nuclease acting in piRNA biogenesis. Here we describe the crystal structure of Zucchini from Drosophila melanogaster and show that it is very similar to the bacterial endonuclease, Nuc. The structure also reveals that homodimerization induces major conformational changes assembling the active site. The active site is situated on the dimer interface at the bottom of a narrow groove that can likely accommodate single-stranded nucleic acid substrates. Furthermore, biophysical analysis identifies protein segments essential for dimerization and provides insights into regulation of Zucchini's activity.
\end{abstract}

Keywords: Zucchini; piRNA; Piwi; nuclease; phospholipase; PLD6; MitoPLD

\section{INTRODUCTION}

Piwi-interacting RNAs (piRNAs) are $\sim 30$-nucleotide (nt) long small RNAs that associate with the Piwi clade of small RNA-binding proteins called Argonautes. A universal role for the piRNA pathway is to silence transposon elements in animal gonads (Ghildiyal and Zamore 2009; Malone and Hannon 2009; Siomi et al. 2011). piRNAs have been shown to derive from discrete genomic regions called piRNA clusters, transposon transcripts, and certain mRNAs, but the mechanism of their biogenesis is still not clear. Small RNAs like microRNAs and small interfering RNAs (siRNAs) utilize double-stranded RNA precursors that are processed by the RNase III enzyme Dicer. In contrast, biogenesis of piRNAs is independent of Dicer (Vagin et al. 2006; Houwing et al. 2007), suggesting that they likely originate from single-

\footnotetext{
${ }^{4}$ Corresponding authors

E-mail orsolya.barabas@embl.de

E-mail pillai@embl.fr

Article published online ahead of print. Article and publication date are at http://www.rnajournal.org/cgi/doi/10.1261/rna.034967.112.
}

stranded (ss) RNAs (Brennecke et al. 2007). Our current understanding of piRNA biogenesis suggests a two-stage process via a primary and a secondary processing pathway (Brennecke et al. 2007). Primary processing is still very ambiguous with unknown nuclease(s) implicated in liberating 30-nt piRNAs from long, single-stranded precursors to generate piRNAs with prominently uridine at position 1 (1U). In secondary processing, primary piRNA-guided Piwi endonuclease action on target RNAs generates $5^{\prime}$ ends of new secondary piRNAs that feed into a piRNA amplification cycle (Brennecke et al. 2007; Gunawardane et al. 2007). All genetically and biochemically identified factors of piRNA biogenesis appear to gather with the Piwi proteins in unique perinuclear, cytoplasmic granules called the nuage, which are considered to be sites of piRNA biogenesis and action (Aravin et al. 2009; Lim et al. 2009; Siomi et al. 2011).

Drosophila Zucchini (dZuc) or its mouse ortholog MitoPLD (hereafter referred to as mZuc) have been shown to be essential components of the piRNA pathway, with a likely role in primary biogenesis (Pane et al. 2007; Malone et al. 2009; Saito et al. 2009; Haase et al. 2010; Huang et al. 2011; 
Watanabe et al. 2011). They show amino acid sequence homology with members of the phospholipase D (PLD) family. In addition to phospholipases, this widespread protein family includes enzymes with diverse functions, like cardiolipin synthases, phosphatidylserine synthases (PSS), pox viral envelope proteins, and some bacterial nucleases. Existing biochemical data supports mZuc being a phospholipase that cleaves the mitochondria-specific lipid cardiolipin to generate phosphatidic acid (PA) (Huang et al. 2011; Watanabe et al. 2011). Since PA has been shown to facilitate aggregation of mitochondria in cell culture, mZuc was suggested to regulate formation of the perinuclear nuage that hosts piRNA biogenesis. However, some PLD members are proven nucleases like the bacterial DNA endonuclease Nuc (Pohlman et al. 1993; Stuckey and Dixon 1999) from Salmonella typhimurium. While most PLD family members are bi-lobal proteins with two copies of the catalytic motif $\left(\mathrm{HxKx}_{4} \mathrm{Dx}_{6} \mathrm{GSxN}\right)$, Nuc carries a single catalytic motif and functions in a homodimeric form (Stuckey and Dixon 1999). Sequence alignments suggest that Zuc is more similar to Nuc than to other PLD family members with phospholipase activity (Fig. 1A), which appears to contradict its previously implicated lipase function.

Here we have determined the crystal structure of $\mathrm{dZuc}$ and show that it remarkably resembles Nuc. Furthermore, we show that, similarly to Nuc, Zuc also dimerizes, which induces conformational changes, assembling the active site. We identify protein residues required for dimerization and model the dimeric structure based on Nuc. Comparison with Nuc and a PLD from Streptomyces (Leiros et al. 2004) suggests that the active site of Zuc is more likely to bind single-stranded nucleic acids than phospholipids.

\section{RESULTS AND DISCUSSION}

$\mathrm{dZuc}\left(89-253\right.$ aa) crystallized in space group $\mathrm{P} 2{ }_{1}$, and the structure was refined to $\mathrm{R}_{\text {work }}=21.85 \%$ and $\mathrm{R}_{\text {free }}=25.54 \%$ (Table 1 and Protein Data Bank [PDB] 4H4A). The structure reveals a monomer with an overall fold that is remarkably similar to Nuc (RMSD $1.67 \AA$ over $123 \mathrm{C} \alpha$-s) (Figs. 1B, $2 \mathrm{~A}, \mathrm{~B})$. Detailed comparison of Nuc and dZuc structures reveals two major differences. First, our Zuc structure lacks the N-terminal $\beta$-strand of Nuc (marked dark red in Fig. 2A). Second, two loops (162-171 and 202-220, yellow in Fig. 2B) that carry putative active site residues are misplaced and partially disordered in the dZuc structure. As a result, the active site residues turn away from each other and do not form a compact active site. This is in sharp contrast with Nuc, where the catalytic residues all assemble around the putative binding site of the scissile phosphate (Fig. 2A).

Nuc forms a homodimer in its catalytically competent form (Stuckey and Dixon 1999). Dimerization is mediated by polar and H-bonding interactions mainly between the catalytic residues, as well as by extended hydrophobic interactions involving several residues in helix $\alpha 5$ and strand $\beta 1$. The overall structural similarity of dZuc with Nuc, together with the conservation of most amino acids involved in subunit contacts, suggests that dZuc also dimerizes to exhibit its function. In fact, the N-terminal $\beta$-strand $(\beta 1)$ 
TABLE 1. Crystallographic data statistics

\begin{tabular}{|c|c|}
\hline & dZuc(89-253 аa) \\
\hline \multicolumn{2}{|l|}{ Data } \\
\hline Space Group & $\mathrm{P} 2_{1}$ \\
\hline Unit cell $\left(\AA{ }^{\circ}{ }^{\circ}\right)$ & $\begin{array}{l}a=35.64, b=51.28 \\
c=40.97, \beta=107.6\end{array}$ \\
\hline Wavelength $(\AA)$ & 0.97942 \\
\hline Resolution $(\AA)$ & $40-2.2(2.26-2.20)$ \\
\hline R-sym (\%) & $7.2(54.4)$ \\
\hline Rmrgd-F (\%) & $14.2(80.0)$ \\
\hline $1 / \sigma$ & $11.4(2.3)$ \\
\hline Completeness (\%) & $99.6(99.6)$ \\
\hline Redundancy & $3.5(3.2)$ \\
\hline Total observations & $48,633(3350)$ \\
\hline Unique reflections & $14,007(1045)$ \\
\hline Wilson B & 43.3 \\
\hline \multicolumn{2}{|l|}{ Phasing } \\
\hline CC (SHELXD) & 41.6 \\
\hline FOM (DM) & 0.606 \\
\hline \multicolumn{2}{|l|}{ Refinement } \\
\hline Resolution $(\AA)$ & $40-2.2$ \\
\hline Reflections, working set & 12,619 \\
\hline R-work (\%) & 21.9 \\
\hline Reflections, test set & 1383 \\
\hline R-free $(\%)$ & 25.5 \\
\hline RMSD bond $(\AA) /$ angle $\left(^{\circ}\right)$ & $0.003 / 0.67$ \\
\hline RMSD bonded B & 3.5 \\
\hline
\end{tabular}

of Nuc (Fig. 2A) that is missing from the dZuc structure (Fig. 2B) is an integral part of the dimerization interface, and its absence in our dZuc construct may have resulted in failure to crystallize in dimeric form. Since the structural equivalent of this $\beta$-strand was not straightforward to identify in Zuc, we investigated this question further by analyzing the oligomeric state of various N-terminally truncated dZuc (data not shown) and mZuc constructs. Analytical ultracentrifugation and size-exclusion chromatography results show that only $\mathrm{mZuc}(36-212 \mathrm{aa})$ forms a stable dimer (Figs. 2C,D), suggesting that residues 36-44 of mZuc are part of the dimer interface and are essential for dimerization.

To visualize the dimeric conformation of Zuc, we created a model from our dZuc structure based on the dimeric structure of Nuc (Fig. 2A,B). This model reveals that most of the dimer interface is structurally conserved in $\mathrm{dZuc}$ to the amino acid level, and it is likely that dZuc will dimerize in a similar way to Nuc. Interestingly, the novel conformations of loops 162-171 and 202-220 found in the dZuc structure are incompatible with dimerization, as they result in clashes between residues 169-170 from one subunit and 217-218 from the other subunit as well as between residues 204 from the two subunits (Fig. 2B). Since these segments are flexible (partly disordered in our structure) and quite well conserved between Nuc and Zuc (Fig. 1A), it is likely that their conformation will change significantly upon dimerization and they will acquire stable ordered conformation similar to those seen in Nuc. Such dimerization-induced ordering and assembly of active site residues would provide a clever regulatory mechanism that is also used by other nucleases (Barabas et al. 2008; Rice and Correll 2008; Smits et al. 2009) to prevent futile processing of cellular nucleic acids.

Biochemical data have previously suggested that Zuc is a phospholipase with activity against cardiolipin, while amino acid and structural homology would rather support a nuclease function. To further investigate the possible functions of $\mathrm{Zuc}$ we have analyzed the electrostatic potential at the molecular surfaces of Nuc and Zuc. For this analysis we used a dZuc dimer model that we created from our crystal structure modeling both dimerization and the conformation of the active site loops based on the Nuc structure (Fig. 3A-C). Intriguingly, the molecular surface of both Nuc and Zuc reveals an elongated positively charged groove at the subunit interface in the dimer (Fig. 3A,D). Close to the center of this groove we find the catalytic His residues that were proposed to act as nucleophiles in phosphodiester bond cleavage in all PLD family enzymes (Stuckey and Dixon 1999). The size and shape of the groove in the Nuc protein shows that it can easily accommodate a double-stranded nucleic acid, a natural substrate of Nuc (Fig. 3D). On the other hand, the surface groove of $\mathrm{Zuc}$ is much narrower and moderately charged (Fig. 3A). It can hardly accommodate double-stranded nucleic acids, but it may be well suitable to accept single-stranded DNA or RNA.

Interestingly, the electrostatic potential surface of a canonical PLD enzyme from Streptomyces (Leiros et al. 2004) reveals that the active site of that enzyme is located at the bottom of a narrow cavity, which is bordered with negative charges (Fig. 3E). This is in contrast with Nuc and Zuc, both of which feature an elongated positively charged surface groove. These observations support that, similarly to Nuc, Zuc might have a nuclease activity toward singlestranded nucleic acids. However, as shown by previous studies Zuc may act on phospholipids as well. A dual activity toward RNA and cardiolipin could uniquely link nuage formation and piRNA processing and may thus provide an attractive regulatory mechanism for the piRNA pathway.

\section{MATERIALS AND METHODS}

\section{DNA constructs}

The gene for D. melanogaster Zucchini (NCBI Reference sequence NP_609530) was kindly provided by Attilio Pane and Trudi Schupbach (Pane et al. 2007) and Mus musculus Zucchini (SwissProt: Q5SWZ9) was PCR-amplified from a mouse testis cDNA library. Both genes were cloned into pETM-22 expression vector (a modified pET-24d, provided by the EMBL Protein Expression and Purification Core Facility-EMBL PepCore).

\section{Expression and purification}

All constructs were expressed with an N-terminal ThioredoxinAtag followed by a $\mathrm{His}_{6}$-tag. Expression was performed in Escherichia coli RosettaII(DE3)* cells at $16^{\circ} \mathrm{C}$ for $18 \mathrm{~h}$. 
A

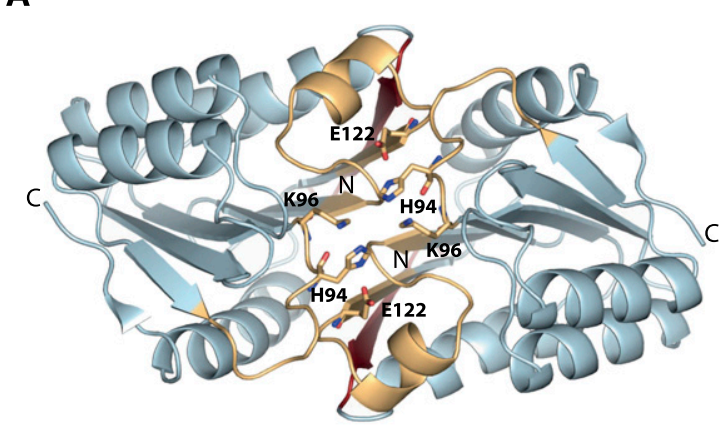

Nuc dimer in active conformation

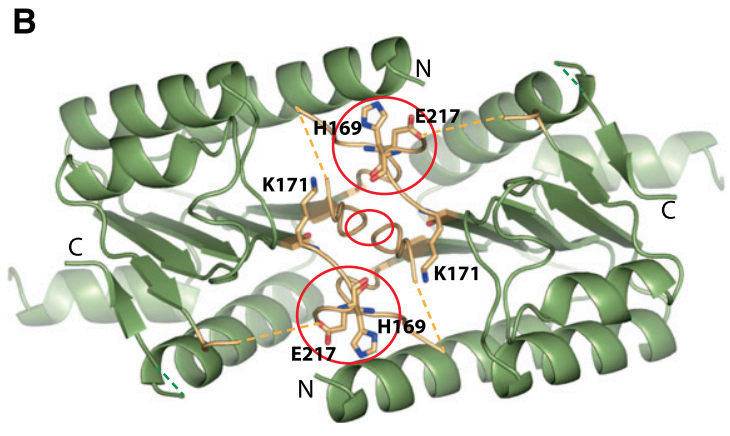

Modelled dZuc dimer in inactive conformation

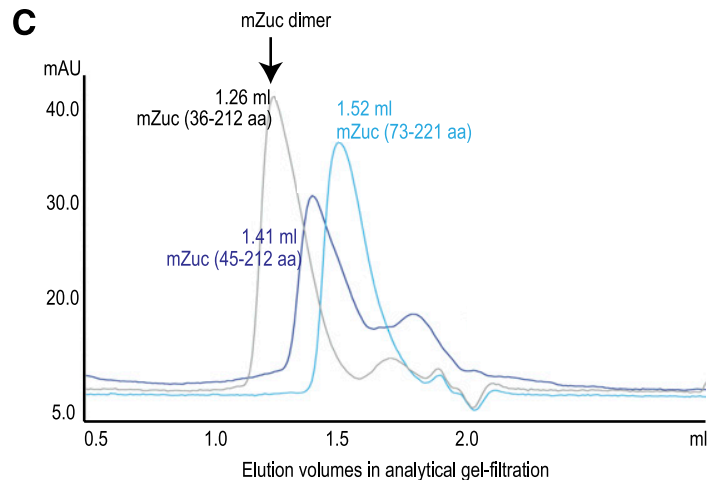

D

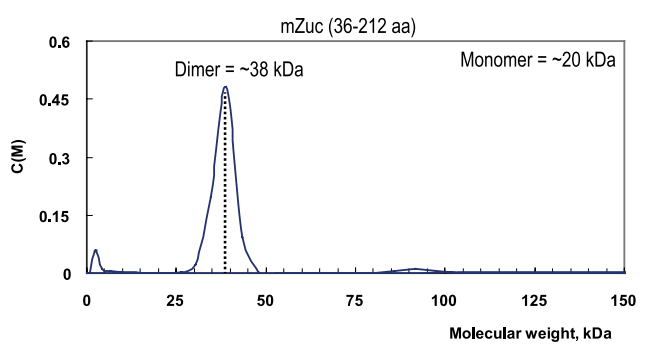

Analytical ultracentrifugation analysis of mZuc

FIGURE 2. Zucchini forms homodimers. (A) Crystal structure of dimeric Nuc (PDB 1BYR) (Stuckey and Dixon 1999). The N-terminal $\beta$-strand (dark red) is required for dimerization. The active site loops (yellow) are in their catalytically competent conformation. (B) dZuc dimer modeled on the related bacterial nuclease Nuc dimer structure. The active site loops (yellow) are misplaced and disordered resulting in clashes on the dimer interface (red circles). (C) Gel-filtration chromatograms for three mouse Zucchini (mZuc) constructs. Dimer formation, as determined by elution volumes (in milliliters), is seen only with the construct mZuc(36-221 aa). (D) Analytical ultracentrifugation analysis of mZuc shows that it sediments as a dimer.

\section{$d Z u c(89-253)$}

The fusion protein was purified by $\mathrm{Ni}^{2+}$-NTA chromatography following the manufacturer's instructions (GE Healthcare). The eluate was dialyzed to an imidazole-free buffer and incubated with PreScission protease (EMBL PepCore) overnight at $4^{\circ} \mathrm{C}$. ThioredoxinA-His ${ }_{6}$-tag and uncleaved fusion protein were removed by a second affinity purification step. The protein was further purified by size-exclusion chromatography using a Superdex $20016 / 60$ column (GE) and stored in $50 \mathrm{mM}$ Tris- $\mathrm{HCl} \mathrm{pH}$ 8.0, $500 \mathrm{mM} \mathrm{NaCl}, 5 \%$ glycerol, $0.1 \mathrm{mM}$ tris(2-carboxyethyl) phosphine (TCEP).

The selenomethionine (SeMet) derivative of $\mathrm{dZuc}(89-253)$ was expressed in E. coli B834(DE3) cells that were grown in M9-minimal medium supplemented with $50 \mathrm{mg} / \mathrm{L} \mathrm{L-SeMet}$ (Sigma). Expression was induced with $0.5 \mathrm{mM}$ IPTG and cultures were grown for $19 \mathrm{~h}$ at $16^{\circ} \mathrm{C}$. The derivative protein was purified using the same procedure as the wild-type protein.

$m Z u c(36-212), m Z u c(45-212), m Z u c(73-221)$

Purification of all mZuc constructs was performed as described for $\mathrm{dZuc}(89-253)$ and differed only in the buffer used (1× PBS, 1 $\mathrm{M} \mathrm{NaCl}, 5 \%$ glycerol, $0.1 \mathrm{mM}$ TCEP).

\section{Crystallization and data collection}

Crystals of dZuc(89-253) and its SeMet derivative were obtained within $1 \mathrm{wk}$ at $20^{\circ} \mathrm{C}$ using the vapor diffusion method in hanging drops; $3 \mathrm{mg} / \mathrm{mL}$ protein solutions were mixed at 1:1.5-2 ratios with reservoir solutions containing 10\%-15\% PEG 3350 and $0.1 \mathrm{M}$ BisTris pH 6.0 and followed by micro-seeding. Crystals were cryo-protected prior to data collection by transferring them to mother liquor containing 12\% 2,3-butanediol (v/v) and flashfreezing in liquid $\mathrm{N}_{2}$. X-ray diffraction data were collected from native crystals on beamline ID14-4 at ESRF (Grenoble, France) to $2.2 \AA$ resolution and a three-wavelength anomalous data set was collected on SeMet-derivative crystals at beamline PXIII at SLS (Villigen, Switzerland) to the same resolution. Crystals of $\mathrm{dZuc}(89-253)$ belong to space group $\mathrm{P} 2_{1}$ and contain one monomer per asymmetric unit.

\section{Structure determination and refinement}

Data sets were processed and scaled using XDS and XSCALE (Kabsch 2010). The structure of $\mathrm{dZuc}$ (89-253) was solved using Auto-Rickshaw (Panjikar et al. 2005), and the best phases were obtained by single-wavelength anomalous diffraction method with SHELX (Sheldrick 2008) using a data set collected at the anomalous peak of selenium. The good quality experimental phases (FOM 
A

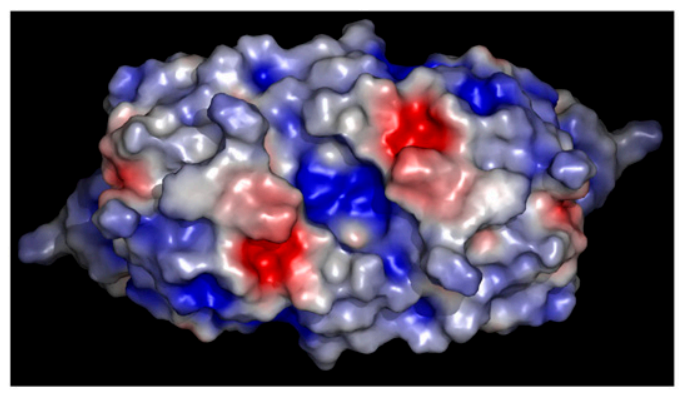

dZuc

B

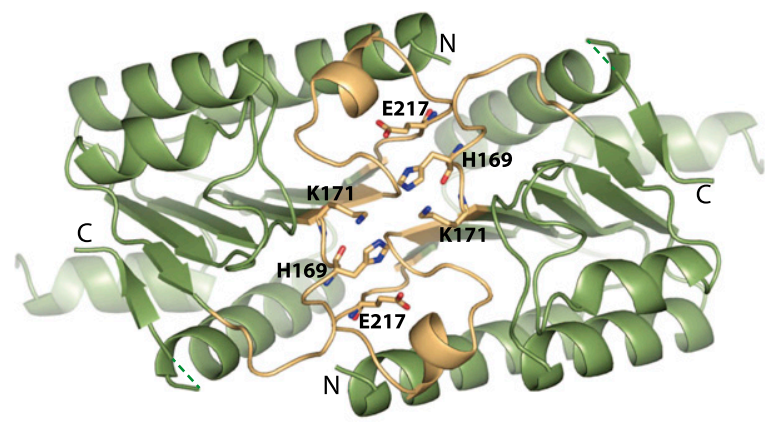

Modelled dZuc dimer in active conformation

C

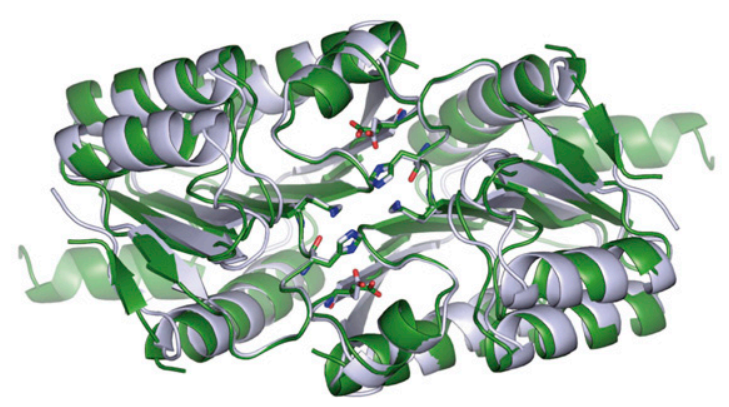

D

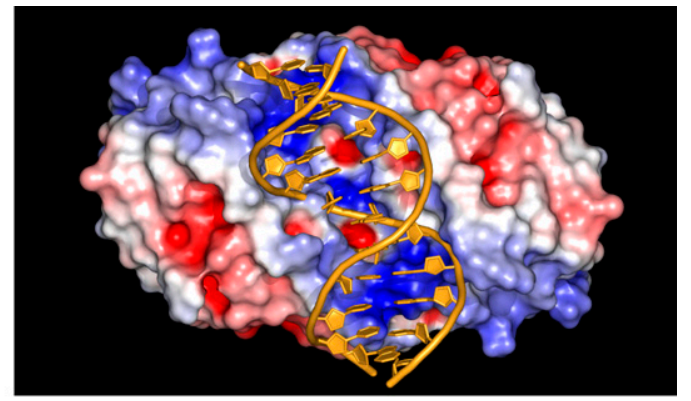

Nuc

E

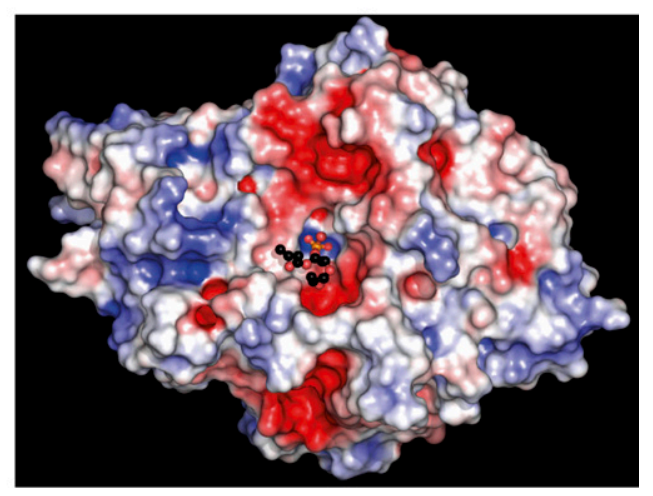

PLD from Streptomyces

FIGURE 3. Zucchini dimer probably accepts ssRNA substrates. (A) Surface representation of dZuc dimer colored by electrostatic surface potential (blue, positive; red, negative). The positively charged groove with catalytic residues is too narrow to accommodate a double-stranded nucleic acid. (B) Ribbon representation is shown for the dZuc dimer model that was used in $A$. It was created based on the related bacterial nuclease Nuc dimer structure, with the active site loops (yellow) and catalytic residues (stick representation) modeled in the catalytically competent state. $(C)$ Overlay of ribbon representation of Nuc dimer (gray) and modeled dZuc dimer (green). (D) Surface representation of Nuc dimer with a double-stranded DNA (orange) modeled into the positively charged groove. (E) Surface representation of a PLD from Streptomyces (PDB 1V0Y) (Leiros et al. 2004) showing electrostatic surface potential (blue, positive; red, negative). The product phospholipid is shown in balland-stick representation highlighting the location of the enzyme active site.

0.606) allowed automatic building of $43 \%$ of the model in Arp/ warp (Perrakis et al. 1999) following density modification with DM (Cowtan 1994). This initial model was then refined through iterative cycles of manual model building in COOT (Emsley and Cowtan 2004) and Cartesian simulated annealing, positional, TLS, and restrained B factor refinement using CNS 1.1 (Brünger et al. 1998) and PHENIX (Adams et al. 2010). All molecular figures were made with PyMOL (DeLano 2002).

\section{Modeling}

The dZuc dimer model was created by superposing the monomer structure onto either of the subunits of the Nuc dimer. Subsequently, the dZuc sequence of the active site loops (residues 162-171 and 202-220) was threaded onto the corresponding residues from the Nuc structure and their conformations were optimized using CNS 1.1 to avoid clashing and unfavorable configuration. 


\section{Sedimentation velocity}

The oligomeric state of $\mathrm{mZuc}(36-212)$ was investigated in $1 \times$ PBS, $1 \mathrm{M} \mathrm{NaCl}, 5 \%$ glycerol, $0.1 \mathrm{mM} \mathrm{TCEP}$ buffer at $4^{\circ} \mathrm{C}$ by monitoring its sedimentation properties at $280 \mathrm{~nm}$ using $40,000 \mathrm{rpm}$ in a Beckman Optima XL-A centrifuge fitted with a four-hole AN-60 rotor and double-sector Epon centerpieces. Molecular weight distributions were determined by the C(s)method (Schuck 2000).

\section{Analytical size-exclusion chromatography}

Experiments were performed in $1 \times \mathrm{PBS}, 1 \mathrm{M} \mathrm{NaCl}, 5 \%$ glycerol, $0.1 \mathrm{mM}$ TCEP buffer using a Superdex $753.2 / 30$ column on an Aekta Purifier.

\section{Protein sequence alignments}

Amino acid sequence alignments of $\mathrm{dZuc}, \mathrm{mZuc}$, and the bacterial nuclease (Nuc) were created using ClustalW and manually edited to match the structural superposition of dZuc and Nuc. Secondary structure elements have been predicted using PSI-PRED for protein segments where crystal structures are not available.

\section{DATA DEPOSITION}

The structural coordinates of dZuc have been deposited in the Protein Data Bank (PDB) under the accession code 4H4A.

\section{ACKNOWLEDGMENTS}

We thank Anne-Claude Gavin and Kenji Maeda for fruitful discussions. We thank the Protein Expression and Purification Core Facility for materials and Vladimir Rybin for analytical ultracentrifugation experiments, High-throughput Crystallization facilities at EMBL Grenoble and Heidelberg, and ESRF and SLS for X-ray diffraction data collection. We also thank Cecilia Zuliani for technical assistance. This work was supported by funding from an ERC Starting Grant from EU to R.S.P. (pisilence). Research in O.B. and R.S.P. labs is supported by the EMBL.

Received June 16, 2012; accepted September 14, 2012.

\section{REFERENCES}

Adams PD, Afonine PV, Bunkóczi G, Chen VB, Davis IW, Echols N, Headd JJ, Hung LW, Kapral GJ, Grosse-Kunstleve RW, et al. 2010. PHENIX: A comprehensive Python-based system for macromolecular structure solution. Acta Crystallogr D Biol Crystallogr 66: 213-221.

Aravin AA, van der Heijden GW, Castaneda J, Vagin VV, Hannon GJ, Bortvin A. 2009. Cytoplasmic compartmentalization of the fetal piRNA pathway in mice. PLoS Genet 5: e1000764. doi: 10.1371/ journal.pgen.1000764.

Barabas O, Ronning DR, Guynet C, Hickman AB, Ton-Hoang B, Chandler M, Dyda F. 2008. Mechanism of IS200/IS605 family DNA transposases: Activation and transposon-directed target site selection. Cell 132: 208-220.

Brennecke J, Aravin AA, Stark A, Dus M, Kellis M, Sachidanandam R, Hannon GJ. 2007. Discrete small RNA-generating loci as master regulators of transposon activity in Drosophila. Cell 128: 1089-1103.

Brünger AT, Adams PD, Clore GM, DeLano WL, Gros P, GrosseKunstleve RW, Jiang JS, Kuszewski J, Nilges M, Pannu NS, et al.
1998. Crystallography \& NMR System: A new software suite for macromolecular structure determination. Acta Crystallogr D Biol Crystallogr 54: 905-921.

Cowtan K. 1994. 'dm': An automated procedure for phase improvement by density modification. Joint CCP4 and ESF-EACBM Newsletter on Protein Crystallography 31: 34-38.

DeLano WL. 2002. The PyMol Molecular Viewer. DeLano Scientific, San Carlos, CA, USA. http://www.pymol.org

Emsley P, Cowtan K. 2004. Coot: Model-building tools for molecular graphics. Acta Crystallogr D Biol Crystallogr 60: 2126-2132.

Ghildiyal M, Zamore PD. 2009. Small silencing RNAs: An expanding universe. Nat Rev Genet 10: 94-108.

Gunawardane LS, Saito K, Nishida KM, Miyoshi K, Kawamura Y, Nagami T, Siomi H, Siomi MC. 2007. A slicer-mediated mechanism for repeat-associated siRNA $5^{\prime}$ end formation in Drosophila. Science 315: 1587-1590.

Haase AD, Fenoglio S, Muerdter F, Guzzardo PM, Czech B, Pappin DJ, Chen C, Gordon A, Hannon GJ. 2010. Probing the initiation and effector phases of the somatic piRNA pathway in Drosophila. Genes Dev 24: 2499-2504.

Houwing S, Kamminga LM, Berezikov E, Cronembold D, Girard A, van den Elst H, Filippov DV, Blaser H, Raz E, Moens CB, et al. 2007. A role for piwi and piRNAs in germ cell maintenance and transposon silencing in zebrafish. Cell 129: 69-82.

Huang H, Gao Q, Peng X, Choi SY, Sarma K, Ren H, Morris AJ, Frohman MA. 2011. piRNA-associated germline nuage formation and spermatogenesis require MitoPLD profusogenic mitochondrial-surface lipid signaling. Dev Cell 20: 376-387.

Kabsch W. 2010. Integration, scaling, space-group assignment and post-refinement. Acta Crystallogr D Biol Crystallogr 66: 133-144.

Leiros I, McSweeney S, Hough E. 2004. The reaction mechanism of phospholipase D from Streptomyces sp. strain PMF. Snapshots along the reaction pathway reveal a pentacoordinate reaction intermediate and an unexpected final product. J Mol Biol 339: 805-820.

Lim AK, Tao L, Kai T. 2009. piRNAs mediate posttranscriptional retroelement silencing and localization to pi-bodies in the Drosophila germline. J Cell Biol 186: 333-342.

Malone CD, Hannon GJ. 2009. Small RNAs as guardians of the genome. Cell 136: 656-668.

Malone CD, Brennecke J, Dus M, Stark A, McCombie WR, Sachidanandam R, Hannon GJ. 2009. Specialized piRNA pathways act in germline and somatic tissues of the Drosophila ovary. Cell 137: $522-535$.

Pane A, Wehr K, Schupbach T. 2007. zucchini and squash encode two putative nucleases required for rasiRNA production in the Drosophila germline. Dev Cell 12: 851-862.

Panjikar S, Parthasarathy V, Lamzin VS, Weiss MS, Tucker PA. 2005. Auto-Rickshaw: An automated crystal structure determination platform as an efficient tool for the validation of an X-ray diffraction experiment. Acta Crystallogr D Biol Crystallogr 61: 449-457.

Perrakis A, Morris R, Lamzin VS. 1999. Automated protein model building combined with iterative structure refinement. Nat Struct Biol 6: 458-463.

Pohlman RF, Liu F, Wang L, More MI, Winans SC. 1993. Genetic and biochemical analysis of an endonuclease encoded by the IncN plasmid pKM101. Nucleic Acids Res 21: 4867-4872.

Rice PA, Correll CC. 2008. Protein-nucleic acid interactions: Structural biology. Royal Society of Chemistry, Cambridge.

Saito K, Inagaki S, Mituyama T, Kawamura Y, Ono Y, Sakota E, Kotani H, Asai K, Siomi H, Siomi MC. 2009. A regulatory circuit for piwi by the large Maf gene traffic jam in Drosophila. Nature 461: 1296-1299.

Schuck P. 2000. Size-distribution analysis of macromolecules by sedimentation velocity ultracentrifugation and Lamm equation modeling. Biophys J 78: 1606-1619.

Sheldrick GM. 2008. A short history of SHELX. Acta Crystallogr A 64: $112-122$. 
Siomi MC, Sato K, Pezic D, Aravin AA. 2011. PIWI-interacting small RNAs: The vanguard of genome defence. Nat Rev Mol Cell Biol 12: 246-258.

Smits C, Chechik M, Kovalevskiy OV, Shevtsov MB, Foster AW, Alonso JC, Antson AA. 2009. Structural basis for the nuclease activity of a bacteriophage large terminase. EMBO Rep 10: 592-598.

Stuckey JA, Dixon JE. 1999. Crystal structure of a phospholipase D family member. Nat Struct Biol 6: 278-284.
Vagin VV, Sigova A, Li C, Seitz H, Gvozdev V, Zamore PD. 2006. A distinct small RNA pathway silences selfish genetic elements in the germline. Science 313: 320-324.

Watanabe T, Chuma S, Yamamoto Y, Kuramochi-Miyagawa S, Totoki Y, Toyoda A, Hoki Y, Fujiyama A, Shibata T, Sado T, et al. 2011. MitoPLD is a mitochondrial protein essential for nuage formation and piRNA biogenesis in the mouse germline. Dev Cell 20: 364375 . 

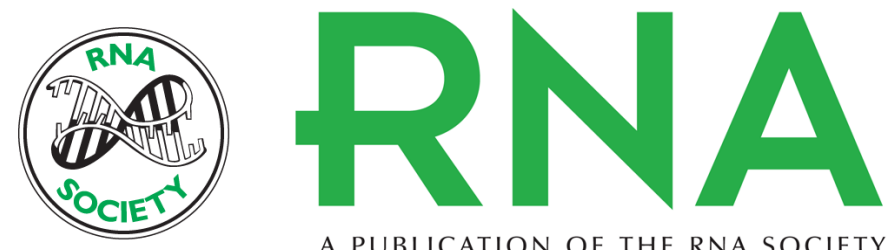

A PUBLICATION OF THE RNA SOCIETY

\section{Crystal structure of the primary piRNA biogenesis factor Zucchini reveals similarity to the bacterial PLD endonuclease Nuc}

Franka Voigt, Michael Reuter, Anisa Kasaruho, et al.

RNA 2012 18: 2128-2134 originally published online October 19, 2012

Access the most recent version at doi:10.1261/rna.034967.112

\section{References This article cites 29 articles, 4 of which can be accessed free at: http://rnajournal.cshlp.org/content/18/12/2128.full.html\#ref-list-1}

Open Access Freely available online through the RNA Open Access option.

License Freely available online through the RNA Open Access option.

Email Alerting Receive free email alerts when new articles cite this article - sign up in the box at the Service top right corner of the article or click here.

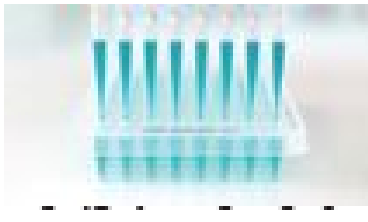

